\title{
The Brain and Spinal Injury Center score: a novel, simple, and reproducible method for assessing the severity of acute cervical spinal cord injury with axial T2-weighted MRI findings
}

\author{
Jason F. Talbott, MD, PhD, ${ }^{1,4}$ William D. Whetstone, MD, ${ }^{2}$ William J. Readdy, BS, ${ }^{3}$ \\ Adam R. Ferguson, PhD, ${ }^{3,4}$ Jacqueline C. Bresnahan, PhD, ${ }^{3,4}$ Rajiv Saigal, MD, PhD, ${ }^{3,4}$ \\ Gregory W. J. Hawryluk, MD, PhD, ${ }^{3,4}$ Michael S. Beattie, PhD, ${ }^{3,4}$ Marc C. Mabray, MD, ${ }^{1}$ \\ Jonathan Z. Pan, MD, PhD, ${ }^{4,5}$ Geoffrey T. Manley, MD, PhD, ${ }^{3,4}$ and Sanjay S. Dhall, MD,4 \\ Departments of ${ }^{1}$ Radiology and Biomedical Imaging, ${ }^{3}$ Neurological Surgery, and ${ }^{5}$ Anesthesia and Perioperative Care, University \\ of California; and ${ }^{2}$ Department of Emergency Medicine and ${ }^{4}$ Brain and Spinal Injury Center, San Francisco General Hospital, \\ San Francisco, California
}

\begin{abstract}
OBJECT Previous studies that have evaluated the prognostic value of abnormal changes in signals on T2-weighted MRI scans of an injured spinal cord have focused on the longitudinal extent of this signal abnormality in the sagittal plane. Although the transverse extent of injury and the degree of spared spinal cord white matter have been shown to be important for predicting outcomes in preclinical animal models of spinal cord injury (SCI), surprisingly little is known about the prognostic value of altered T2 relaxivity in humans in the axial plane.
\end{abstract}

METHODS The authors undertook a retrospective chart review of 60 patients who met the inclusion criteria of this study and presented to the authors' Level I trauma center with an acute blunt traumatic cervical SCI. Within 48 hours of admission, all patients underwent MRI examination, which included axial and sagittal T2 images. Neurological symptoms, evaluated with the grades according to the American Spinal Injury Association (ASIA) Impairment Scale (AIS), at the time of admission and at hospital discharge were correlated with MRI findings. Five distinct patterns of intramedullary spinal cord T2 signal abnormality were defined in the axial plane at the injury epicenter. These patterns were assigned ordinal values ranging from 0 to 4, referred to as the Brain and Spinal Injury Center (BASIC) scores, which encompassed the spectrum of $\mathrm{SCl}$ severity.

RESULTS The BASIC score strongly correlated with neurological symptoms at the time of both hospital admission and discharge. It also distinguished patients initially presenting with complete injury who improved by at least one AIS grade by the time of discharge from those whose injury did not improve. The authors' proposed score was rapid to apply and showed excellent interrater reliability.

CONCLUSIONS The authors describe a novel 5-point ordinal MRI score for classifying acute SCls on the basis of axial T2-weighted imaging. The proposed BASIC score stratifies the SCls according to the extent of transverse T2 signal abnormality during the acute phase of the injury. The new score improves on current MRI-based prognostic descriptions for $\mathrm{SCl}$ by reflecting functionally and anatomically significant patterns of intramedullary $\mathrm{T} 2$ signal abnormality in the axial plane.

http://thejns.org/doi/abs/10.3171/2015.1.SPINE141033

KEY WORDS spinal cord injury; MRI; T2; ASIA; contusion; BASIC; trauma

ABBREVIATIONS AIS = American Spinal Injury Association (ASIA) Impairment Scale; BASIC = Brain and Spinal Injury Center; PACS = picture archiving and communication system; $\mathrm{SCl}=$ spinal cord injury.

SUBMITTED October 6, 2014. ACCEPTED January 21, 2015

INCLUDE WHEN CITING Published online July 10, 2015; DOI: 10.3171/2015.1.SPINE141033.

DISCLOSURE Partial support for this study was provided by a Department of Defense Congressionally Directed Medical Research Programs award (SC140137) to Drs.

Beattie, Manley, Bresnahan, Whetstone, and Ferguson. Dr. Dhall has received speaker honoraria from DePuy and Globus. 
$\mathrm{F}$ OLLOWING the advent and widespread implementation of MRI in the 1980s, many researchers have investigated the prognostic value of MRI findings in assessing acute spinal cord injury (SCI). In particular, the prognostic value of abnormalities in T2-weighted MRI signals has been extensively explored. 3,4,10,13,15,19,27,30,32,42 In the acute phase, a T2 signal abnormality within the injured spinal cord has been attributed to various underlying pathological changes in both human and animal studies. ${ }^{8,25,26,31}$ For example, a T2 hypointense signal reflects the susceptibility-related T2-shortening effect of intracellular deoxyhemoglobin during the acute and subacute phases of hemorrhage. ${ }^{14}$ A T2 hyperintense signal is less specific and probably reflects a combination of vasogenic edema, cytotoxic edema, axonolysis, myelinolysis, inflammatory cellular infiltrate, and petechial hemorrhage..$^{25,28,31}$ Early MRI-based classification systems for acute SCIs defined 3 distinct patterns of intramedullary signal change: Type I, with diffuse T2 hypointensity; Type II, with intramedullary T2 hyperintensity; and Type III, with central T2 hypointensity and a surrounding hyperintense signal..$^{3,10,20}$ Modification of these descriptions in subsequent studies eliminated the Type I pattern because a T2 hypointense hemorrhage was not routinely observed without a significant surrounding T2 hyperintense edema. ${ }^{12,33}$

A more widely adopted classification system defines 4 distinct injury patterns as assessed on a sagittal T2weighted MRI sequence. , $^{1,23,33,35}$ Pattern 1 represents a normal spinal cord signal; Pattern 2 shows a T2 hyperintense intramedullary edema, with its longitudinal extent confined to a single vertebral level; Pattern 3 indicates a multilevel edema; and Pattern 4 includes a mixed hemorrhage and edema. ${ }^{4}$ Such classification systems have been shown to provide measures that correlate with injury severity and that supplement other clinical measures for predicting clinical outcome. ${ }^{1,11,12,23,32,35}$

Patterns based on sagittal T2-weighted MRI signals are most accurate at predicting outcomes when patients have very mild (that is, Pattern 1, indicating a normal cord signal) or severe (Pattern 4, with hemorrhage and edema) injury. ${ }^{4}$ However, in the setting of nonhemorrhagic intramedullary T2 hyperintensity, there is tremendous variability in clinical outcomes. For example, in a metaanalysis, Bozzo et al. reported that among 49 patients presenting with Pattern 3 edema (that is, with multilevel T2 hyperintensity), the injury severity grades of the American Spinal Injury Association (ASIA) Impairment Scale (AIS) were nearly equally distributed at the follow-up: $27 \%$ of these patients had an AIS grade of A, 22\% of B, 24\% of C, and $24 \%$ of D. ${ }^{4}$ This wide variability in outcome data is in part related to the arbitrary measurement of the longitudinal extent of the $\mathrm{T} 2$ signal relative to the height of the vertebral body, in addition to the nonspecific nature of T2 hyperintensity in the spinal cord. Histopathological studies of SCI in animals have revealed that longitudinal measurements do not correlate with functional recovery as well as axial or cross-sectional area does..$^{5}$ In addition, translational studies of axial T2 images in rats have indicated a strong correspondence of axial MRI findings with microscopic histopathology and functional recovery. ${ }^{28}$

Given the limitations of previous longitudinal MRI- based measures of intramedullary signal change and the paucity of axial T2 data on SCIs, we sought to develop a simple and reproducible classification system for blunt traumatic SCI that is based on the transverse extent of intramedullary T2-weighted MRI signal abnormality during the acute phase of injury. We hypothesized that such a classification system would reflect the functionally relevant anatomical distribution of pathological MRI signal changes and therefore yield valuable diagnostic and prognostic information. In this study, we aimed to assess the reliability and validity of this MRI-based classification system in a cohort of patients with blunt traumatic SCIs.

\section{Methods}

\section{Patient Selection}

We performed a retrospective chart review to evaluate the diagnostic and prognostic values of axial T2-weighted MRI findings for rating the severity of acute SCIs in patients admitted to San Francisco General Hospital, a Level I trauma center, between January 2005 and December 2011. This study was approved by the internal review board of the University of California. Patients' records were reviewed in a Department of Neurosurgery database and in cross-referencing trauma logs, with searchable terms and by using electronic medical records (San Francisco, CA). From this database, we retrospectively identified the records of 131 patients who had a principal diagnosis of SCI (codes 953-957 designating discharge diagnoses) according to the International Classification of Diseases, Ninth Revision, Clinical Modification (ICD-9$\mathrm{CM}$ ). Of these patients, 92 had cervical levels of injury, 60 of whom met this study's inclusion criteria.

To be eligible for this study, patients had to be 18 years of age or older; had to have an MRI examination performed within 48 hours of admission which, at a minimum, included T2-weighted images of the cervical spine in both the axial and sagittal planes; and had to have a documented AIS grading performed both at the time of admission and at a follow-up (performed at the time of discharge from the acute-care hospital). We excluded patients younger than 18 years; those with an SCI related to penetrating trauma or with imaging evidence of complete spinal cord transection; and those with MRI studies degraded by motion or other artifacts such that $\mathrm{T} 2$-weighted images were nondiagnostic as assessed by a neuroradiologist (J.F.T.). Patients who underwent surgical decompression, fusion, or both before the MRI examination were also excluded. SCI-trained physiatrists and neurosurgical and neurocritical care attending physicians performed the AIS grading. All eligible patients' AIS grades were obtained within 24 hours of admission and before the MRI examination.

\section{MRI Studies}

All MRI studies were performed on a $1.5 \mathrm{~T}$ GE Genesis Signa scanner (GE Healthcare). Axial T2-weighted fast spin echo imaging was performed with the following parameters (means \pm SDs from 10 randomly selected examinations): TR $3590 \pm 546 \mathrm{msec}$, TE $94.9 \pm 10 \mathrm{msec}$, slice thickness $3 \mathrm{~mm}$, and echo train length $16 \pm 4$. Sagittal T2-weighted fast spin echo imaging was performed 
with the following parameters: TR $3300 \pm 290 \mathrm{msec}$, TE $102 \pm 3 \mathrm{msec}$, slice thickness $3 \mathrm{~mm}$, and echo train length $15 \pm 3$. For both sagittal and axial T2 imaging, the acquisition matrix was $256 \times 256$. The phase encoding direction was left to right for the axial sequences and craniocaudal for the sagittal sequences. The field of view ranged from 16 to $20 \mathrm{~cm}$. Additional sequences performed as part of our routine trauma MRI protocol were not evaluated for the purposes of this study. An axial 2D multiecho recombined gradient echo sequence from a single normal patient was used as a control reference for identifying margins of gray matter at the upper, mid, and lower cervical levels.

\section{Image Analysis and BASIC Scoring}

Axial and sagittal T2-weighted MRI sequences were examined by a fellowship-trained neuroradiologist (J.F.T.) and a spine fellowship-trained neurosurgeon (S.S.D.), who were both blinded to the AIS grade. The epicenter of the SCI was located on the axial T2-weighted sequence and confirmed by cross-referencing with the sagittal T2weighted sequence. A single axial image with the most severe SCI was identified for the scoring. The Brain and Spinal Injury Center (BASIC) scoring was performed according to the observations outlined in Fig. 1. Briefly, an SCI with a BASIC score of 0 represented normal spinal cord $\mathrm{T} 2$ relaxivity without appreciable pathological intramedullary signal. A BASIC score of 1 represented cases in which a pathological T2 hyperintensity was approximately confined to the spinal gray matter (Fig. 2). A BASIC score of 2 was assigned when a pathological intramedullary T2 hyperintensity extended beyond the margins of the central gray matter and obscured the gray-white margins, but did not involve the entire transverse extent of the spinal cord. For these cases, some peripheral normal-appearing white matter was identified. A BASIC score of 3 was assigned when the pathological T2 hyperintensity involved the entire transverse extent of the spinal cord, without any residual normal-appearing white matter. An SCI with a BASIC score of 4 was defined as a BASIC Score 3 injury with additional superimposed discrete foci of intramedullary T2 hypointensity attributed to the presence of macroscopic intramedullary hemorrhage.

The SCIs with BASIC scores of 0,1 , and 2 could be elevated by a single score if a macroscopic hemorrhage was present, although no such cases were identified in our patient cohort. For example, a BASIC score of 2 with the presence of macroscopic hemorrhage would be elevated to BASIC score of 3.

\section{Image Processing}

Digital Imaging and Communications in Medicine (DICOM) images from our university picture archiving and communication system (PACS; Agfa Healthcare) were annotated and cropped for figure production with ImageJ software (available at http://rsb.info.nih.gov/ij and developed by Wayne Rasband at NIH). We produced 3Dcolor surface plots of T2-weighted images with an interactive 3D-surface plot plugin for ImageJ. These surface plots were used only for figure production and were not used for primary image analysis or interrater reliability testing.

\section{Interrater Reliability Testing Protocol}

Interrater reliability was assessed by measuring the mean and SD of scores assigned by multiple raters review-
A

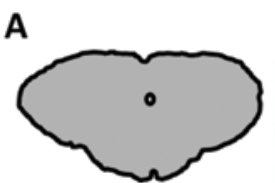

B
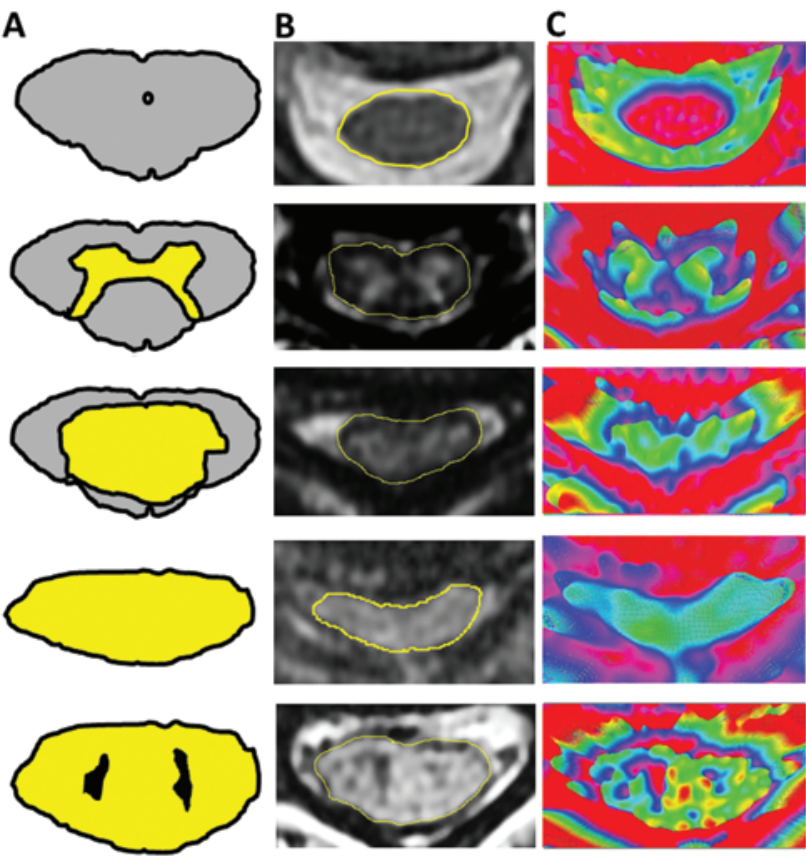
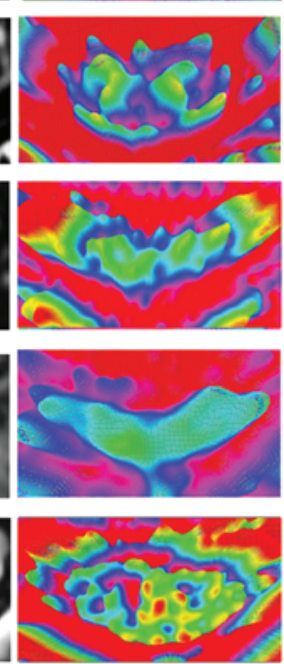

D

BASIC 0: No appreciable intramedullary cord signal abnormality.

BASIC 1: Intramedullary T2 hyperintensity is approximately confined to central gray matter.

BASIC 2: Intramedullary T2 hyperintensity extends beyond expected gray matter margins to involve spinal white matter, but does not involve entire transverse extent of the spinal cord.

BASIC 3: Intramedullary T2 hyperintensity involves entire transverse extent of spinal cord.

BASIC 4: Grade 3 injury plus discrete T2 hypointense foci, consistent with macrohemorrhage.

FIG. 1. The BASIC score of SCIs. Cartoon schematics (A), representative axial T2-weighted MRI scans (B), 3D-color surface plots based on the axial T2 image (C), and brief definitions (D) for each of the 5 BASIC scores (ranging from 0 to 4 ). In the representative MRI scans (B), the external contour of the spinal cord is outlined in yellow for better delineation. Figure is available in color online only. 


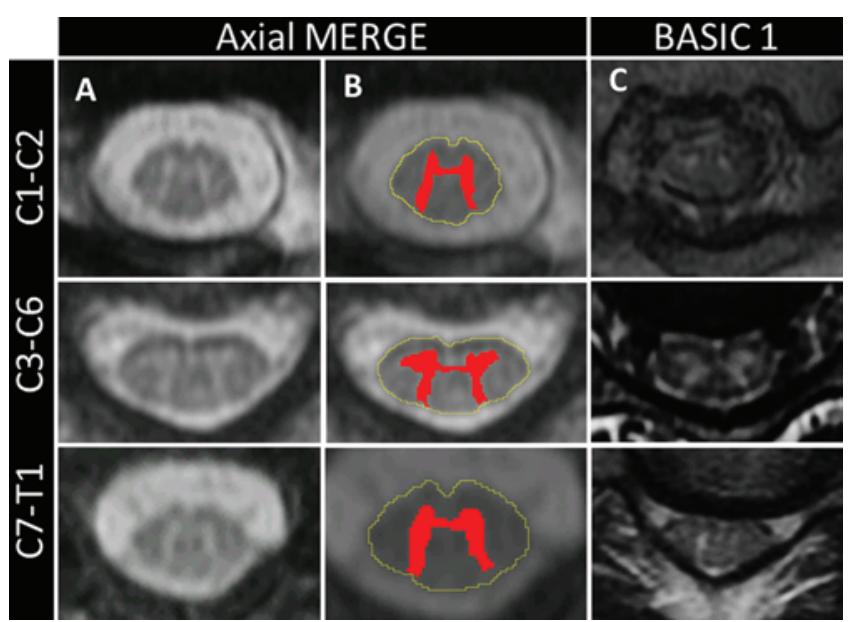

FIG. 2. Familiarity with the normal spinal cord gray matter morphology at rostral (C1-C2), middle (C3-C6), and caudal (C7-T1) cervical vertebral levels is important for rating an SCI as BASIC Score 1. A: Axial multiecho recombined gradient echo (MERGE) image of the normal spinal cord clearly indicates the normal gray matter morphology at the upper, middle, and lower cervical vertebral levels. B: Manual segmentation of cervical spinal cord gray matter based on an axial MERGE images in A with the peripheral cord contour delineated in yellow. Note the large frontal horns related to the cervical enlargement at the C3-6 vertebral levels. C: Axial T2-weighted images from the epicenters of BASIC Score $1 \mathrm{SCls}$ at the upper, middle, and lower cervical vertebral levels. Note that the T2 hyperintensity represents the approximate boundaries of spinal cord gray matter for each cervical level. Figure is available in color online only.

ing 20 MRI studies chosen to represent all parts of the BASIC rating scale; the reliability testing was similar to that in the development of the scale established by Basso, Beattie, and Bresnahan. ${ }^{2}$ Seven participating raters were instructed in the rating during an initial training session in which they were shown MRI studies of a range of SCIs and the method of scoring was explained. The specialties of training of the participants included neuroradiology, neurosurgery, emergency medicine, neuroanatomy, and anesthesiology. The rating of individual images was then practiced in concurrent discussions, followed by each participant silently rating the observations on the MRI studies and then comparing and discussing their scores with those of the instructors.

After the training, each rater was presented with a series of DICOM images including both the injury epicenter and adjacent normal-appearing spinal cord from 20 separate cases from our cohort with SCIs representing all levels of the BASIC scale. The cases were presented in random order. Also provided to each rater were a set of data-recording sheets, an overview of the project background and goals, a set of frequently asked questions with answers, and a score determination guide for ease of assigning scores. All participants then individually examined the 20 images and scored each of them within 20 seconds according to the descriptions provided. The data sheets were then collected, analyzed, and compared with a consensus score for each image, arrived at by the original scale developers' viewing, discussing, and arriving at the consensus score for each image. This consensus score was determined after all raters (including the experienced raters) had completed and submitted their independent ratings of the images.

\section{Statistical Analysis}

All statistical analyses were performed with a commercial software package (SPSS Inc.). Statistical correlation between the BASIC score and AIS grades at both admission and discharge were evaluated with the Pearson correlation coefficient. The differences in BASIC scores among the AIS improvement groups were analyzed with 2-tailed Student t-tests. Statistical significance was determined as $\mathrm{p}<0.05$.

A statistical analysis of the reliability of the BASIC classification system among different observers against the consensus scores was performed with the Kappa co-

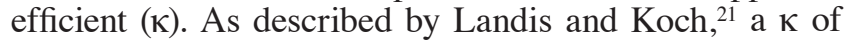
$>0.8$ was interpreted as excellent reliability. The unidimensional nature of the BASIC score was assessed on all cases by all raters with exploratory factor analysis with the principal component extraction method..$^{29,36}$

\section{Results}

\section{Patient Characteristics}

Table 1 shows the demographic and clinical characteristics of our cohort of 60 patients. Table 2 lists the complete admission and discharge AIS data for our entire cohort. All of the SCIs resulted from blunt trauma, and 17 of the patients $(28 \%)$ presented with complete injury (that is, AIS Grade A). The patients were predominantly male (70\%) with a mean age of 56 years (range 18-94 years) (Table 1). The most frequent injury mechanism was fall (53\%), followed by motor vehicle collision (15\%), bicycle accident (10\%), assault (8\%), pedestrian versus automobile accident (7\%), and other or nonspecified mechanism (7\%) (Table 1). The mean length of time between the hospital admission and the spine MRI was $8.6 \pm 6$ hours (range 1-39 hours). The patients were examined at the sole Level I trauma center within a dense urban catchment area where the time from injury to admission at our institution is on average less than 60 minutes. In total, 51 patients

\section{TABLE 1. Characteristics of the patients in this study}

\begin{tabular}{lc}
\hline \multicolumn{1}{c}{ Variable } & All Patients \\
\hline Total no. of patients & 60 \\
\hline Mean age in yrs \pm SD (range) & $56 \pm 20(18-94)$ \\
\hline Sex M/F (\%) & $42 / 18(70 / 30)$ \\
\hline Injury mechanism, no. of patients (\%) & $32(53)$ \\
\hline Fall or jump & $9(15)$ \\
\hline Motor vehicle collision & $6(10)$ \\
\hline Bicycle accident & $5(8)$ \\
\hline Assault & $4(7)$ \\
\hline Pedestrian vs automobile accident & $4(7)$ \\
\hline Other & $8.6 \pm 6(1-39)$ \\
\hline Time to MRI in hrs \pm SD (range) & $23 \pm 24$ \\
\hline Mean time to discharge in days \pm SD
\end{tabular}


TABLE 2. The AIS grades of the 60 patients in this study at admission and at discharge

\begin{tabular}{ccc}
\hline & \multicolumn{2}{c}{ No. of Patients (\%) } \\
\cline { 2 - 3 } AIS Grade & Admission & Discharge \\
\hline A & $17(28)$ & $9(15)$ \\
\hline B & $7(12)$ & $4(7)$ \\
\hline C & $10(17)$ & $10(17)$ \\
\hline D & $18(30)$ & $20(33)$ \\
\hline E & $8(13)$ & $17(28)$ \\
\hline
\end{tabular}

(85\%) underwent an MRI examination within 12 hours of the hospital admission, and only 1 patient (2\%) underwent the examination more than 24 hours after admission. For those patients admitted to the hospital, the average length of hospitalization was 23 days (range 4-128 days).

\section{MRI Findings}

Axial and sagittal T2-weighted MRI sequences indicated intramedullary signal abnormalities in $48(80 \%)$ of the 60 patients. In all patients, 5 distinct patterns of intramedullary signal were identified on the axial $\mathrm{T} 2$-weighted sequence at the injury epicenter (Fig. 1). In 12 patients (20\%), no apparent signal abnormality was observed, and their SCI finding received a BASIC score of 0. In 16 (27\%) of the patients, a T2 signal hyperintensity was observed that largely conformed to the expected morphology of the central spinal gray matter; therefore, these patients' SCI was rated as BASIC Score 1 . In 18 patients (30\%), we observed a pattern of intramedullary $\mathrm{T} 2$ hyperintensity at the injury epicenter that extended beyond and obscured the expected margins of the central gray matter, but did not involve the entire transverse extent of the spinal cord on axial imaging; their injuries were therefore rated BASIC Score 2. In 9 patients (15\%), an SCI resulting in diffuse intramedullary $\mathrm{T} 2$ hyperintensity that involved the transverse extent of the cord was rated as BASIC Score 3 . The remaining 5 patients $(8 \%)$ had SCIs that resulted in diffuse T2 hyperintensity with superimposed discrete foci of T2 hypointensity, consistent with intramedullary hemorrhage, and their SCI severities were rated as BASIC Score 4. None of the patients showed evidence for macroscopic hemorrhage in the absence of diffuse transverse T2 hyperintensity.

\section{BASIC Score Strongly Correlates With Admission AIS Grade}

We observed a highly significant correlation between the AIS grade at the time of admission and the morphological pattern of intramedullary signal abnormality as rated by the BASIC score on the admission MRI study. Figure 3 graphically displays the linear correlation between the AIS grade and the BASIC score at admission. Along the severe spectrum of an acute SCI, a BASIC score of 3 or 4 was nearly always associated with an admission AIS grade of A, that is, in $13(76 \%)$ of the 17 patients with an admission AIS Grade A. Among the 43 patients with an AIS grade less severe than A, only 1 patient (2\%) had

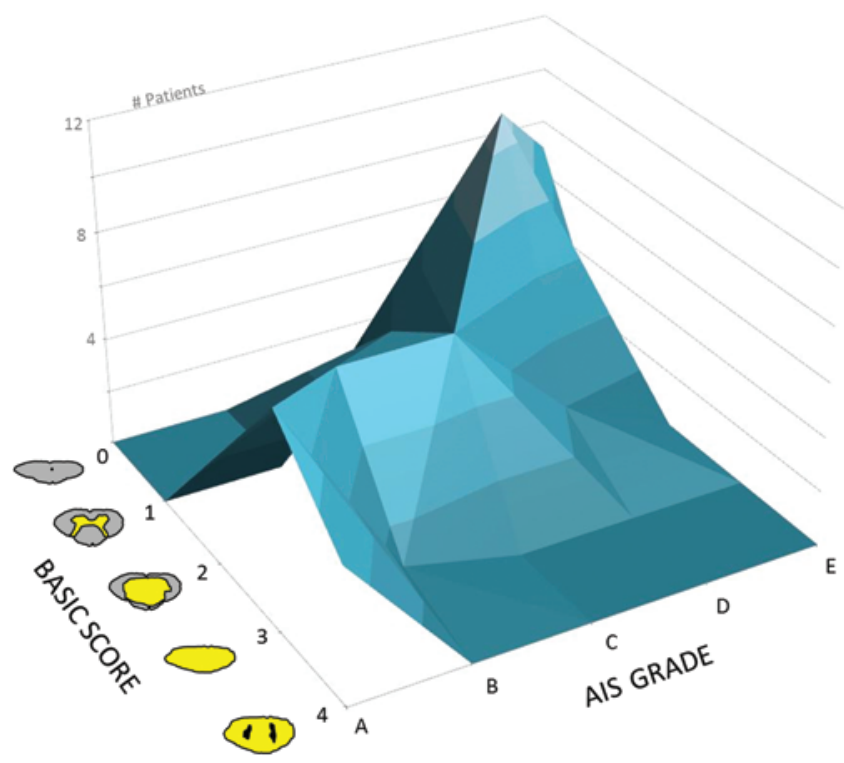

FIG. 3. A 3D surface plot indicates a strong correlation of the BASIC score with the AIS grade at the time of hospital admission (Pearson coefficient $=-0.877, p=4.0 \times 10^{-20}$ ). The height of the surface plot (that is, the z-axis) corresponds to the number of patients with corresponding BASIC scores and AIS grades within our cohort. Note the course of the peak of the surface plot clearly tracing the strong linear correlation between the BASIC score and the AIS grade. Figure is available in color online only.

a BASIC score of 3, and none had a BASIC score of 4 . A BASIC score of 4 was always observed with an SCI rated as AIS Grade A at admission. Thus, a high BASIC score, that is, of 3 or 4 , was specific for severe injury at admission.

On the mild end of the SCI severity spectrum, an SCI with a BASIC score of 1 or 0 was never observed in patients with an AIS grade of A or B on admission. A BASIC score of 0 (that is, a normal cord signal) was entirely limited to patients with an admission AIS grade of D or E.

\section{BASIC Score Strongly Correlates With AIS Grade at Discharge}

The correlation between the AIS grade at the time of discharge and the BASIC score based on the morphological pattern of intramedullary signal abnormality on the admission MRI study was also highly significant. Figure 4 displays the linear correlation between the admission AIS grade at discharge and the BASIC score. Figure 5 shows a plot of the admission and discharge AIS grades for all patients stratified by the 5 BASIC score groups. Of 12 patients with an SCI rated as BASIC Score 0, $11(92 \%)$ were discharged with an AIS Grade E, with the remaining single patient discharged with AIS Grade D. All 16 patients with a BASIC score of 1 were discharged with an AIS grade of D or E. Of 18 patients with a BASIC score of $2,16(88 \%)$ were discharged with an AIS grade of C or D. Among 9 patients with a BASIC score of 3, 6 (67\%) were discharged with an AIS grade of A or B. All 4 patients with a BASIC score of 4 were discharged with an AIS grade of $\mathrm{A}$. 


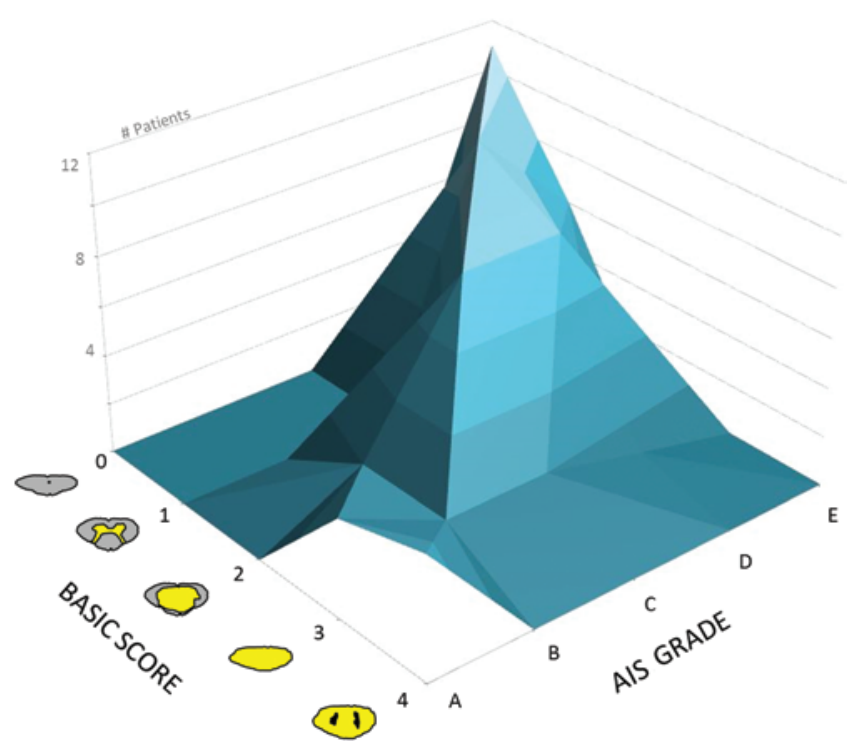

FIG. 4. A 3D surface plot indicates a strong correlation between the BASIC score and the AIS grade at the time of hospital discharge (Pearson coefficient $=-0.880, p=2.0 \times 10^{-20}$ ). The height of the surface plot (that is, the z-axis) corresponds to the number of patients with corresponding BASIC scores and AIS grades within our cohort. Note the course of the peak of the surface plot clearly tracing the strong linear correlation between the BASIC score and the AIS grade. Figure is available in color online only.

\section{BASIC Score Distinguishes Patients With an Admission AIS Grade of A Who Improve at Discharge}

At the time of discharge, 8 (47\%) of the 17 patients with an admission AIS grade of A improved by at least one AIS grade. The BASIC scores among AIS Grade A patients whose condition did not improve were significantly higher than among those who did improve by at least one AIS grade $(3.6 \pm 0.5$ vs $2.6 \pm 0.5$, respectively, $\mathrm{p}<0.01$; Fig. 6).

\section{BASIC Score and Interobserver Reliability}

The mean and median $\kappa$ scores for all raters were 0.83 and 0.81 , respectively (both $\mathrm{p}<0.00001$ ), relative to the consensus score, consistent with excellent reliability and reproducibility. A factor analysis with principal component analysis indicated that the BASIC score represented a unidimensional outcome, with high correspondence among the 7 raters (Table 3 and Fig. 7).

\section{Discussion}

In the present study, we sought to classify the severity of an acute SCI according to the transverse extent of signal abnormalities as qualitatively assessed on a single axial T2-weighted MR image centered at the lesion epicenter. Specifically, we introduce a 5-point (ranging from 0 to 4) ordinal classification system, which encompasses the spectrum of SCI severity, from a normal-appearing spinal cord to a diffusely abnormal cord signal hyperintensity with superimposed macroscopic intramedullary hemorrhage (Fig. 1). We excluded cord transection injuries from consideration because of the distinct and easily distinguished imaging pattern associated with this SCI type. The proposed BASIC score builds on previously described MRIbased systems for classifying acute traumatic SCIs, and in our analyses it strongly correlated with AIS grades at the hospital admission for the SCI and at discharge (Figs. 3 and 4). Moreover, the BASIC score stratifies the SCIs on the basis of the anatomically and functionally relevant extent of transverse injury. It may help identify those patients who present with the most severe clinical injury (that is, with AIS Grade A) and who will improve by at least one AIS grade by the time of discharge (Fig. 6).

Both human and animal studies have demonstrated that the transverse extent of an SCI and relative white matter sparing are major determinants of functional outcomes. $5,6,16,18,22$ To our knowledge, the present study is the first to correlate clinical symptoms and outcomes with the

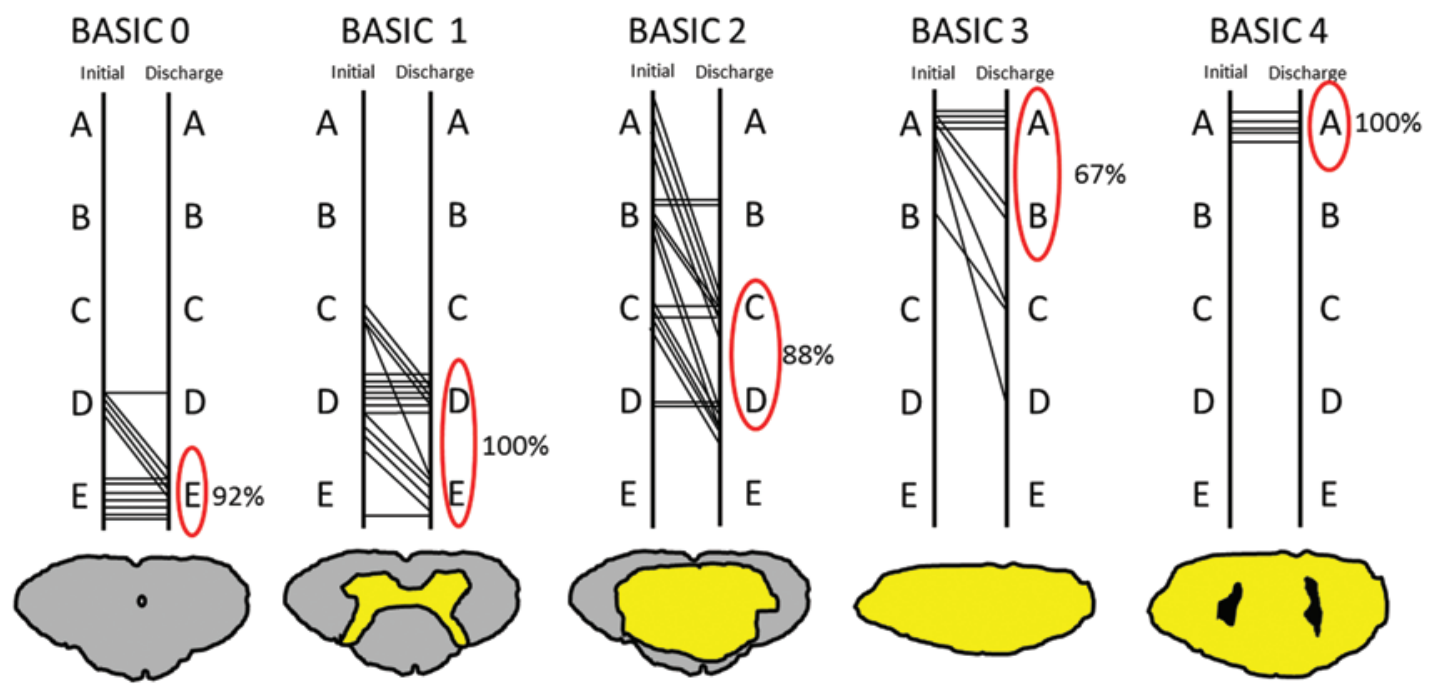

FIG. 5. Admission and discharge AIS grades for all patients in our cohort are plotted within each BASIC score group, with a cartoon schematic of the SCI below each plot. The percentages of patients within each BASIC group with a discharge AIS grade circled in red are listed to the right of the discharge AIS grades. Figure is available in color online only. 

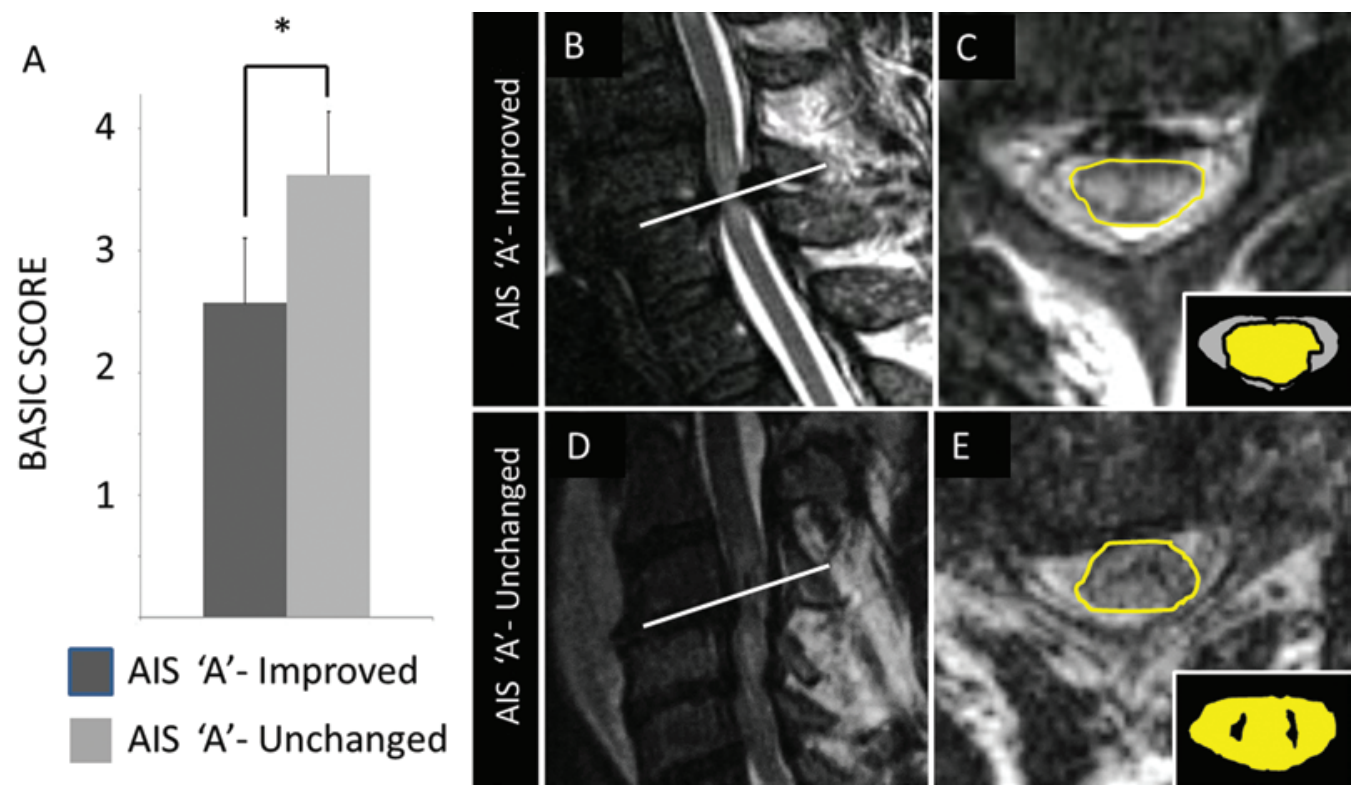

FIG. 6. The BASIC scores for patients who presented with complete injury (that is, with AIS Grade A) and who improved by at least one AIS grade are significantly lower than those for AIS Grade A patients whose SCl showed no improvement. The bar graph shows a significantly lower BASIC score for patients with AIS Grade A whose injury improved in AIS grade by the time of follow-up compared with AIS Grade A patients whose injuries did not improve $(p<0.01)(\mathbf{A})$; error bars indicate the SD. Sagittal (B) and axial (C) T2-weighted images from a patient with an SCl sustained in a fall and presenting with AIS Grade A indicate abnormal intramedullary T2 hyperintensity with a pattern of T2 signal abnormality on the axial image at the injury epicenter (B) consistent with a BASIC score of 2 (see schematic inset in the right lower corner). This patient's condition improved to AIS Grade $\mathrm{C}$ at the follow-up. Sagittal (D) and axial (E) T2-weighted images from a patient with an SCl injury due to an assault and also presenting with AIS Grade A show abnormal intramedullary T2 signal at the injury epicenter (D) consistent with a BASIC score of 4 (see schematic inset in the right lower corner). This patient did not recover from the SCl at the time of follow-up. White lines in B and $\mathrm{D}$ approximate the level of the axial T2 image for each patient. For better delineation, the peripheral margins of the spinal cord are outlined in yellow in $\mathrm{C}$ and $\mathrm{E}$. Figure is available in color online only.

transverse extent of MRI T2 signal abnormality in the axial plane in humans. Rather than arbitrary measurements of the longitudinal extent of signal abnormality in the sagittal plane, axial imaging enables the definition of anatomically relevant spinal involvement in a graded manner. With an SCI severity rated as BASIC Score 1, T2 hyperintensity is approximately confined to the spinal gray matter. The relatively good clinical outcomes at discharge for patients with a BASIC score of 1 in our study (all of these patients were discharged with an AIS grade of D or E) suggest such signal abnormality does not reflect significant coagulative necrosis or irreversible frontal horn disruption, but more likely represents vasogenic edema, as has been suggested by other authors. ${ }^{9,31}$

When a T2 hyperintense signal extended beyond the approximate confines of gray matter (that is, in patients with BASIC scores of 2-4), patients had a worse prognosis (Fig. 5). Importantly, distinguishing patients who have some spared white matter signal (a BASIC score of 2) from those with diffuse transverse $T 2$ hyperintensity (a BASIC score of 3) allows for identifying those patients whose SCIs would all be classified as having multilevel hyperintensity according to previous sagittal T2 signal grading systems. ${ }^{4}$ Our observations of a functionally relevant distinction between SCIs rated as BASIC Score 2 or 3 are consistent with preclinical data, and this corroboration highlights the important role of spared white matter in predicting outcomes. ${ }^{6,17,18,22}$ In our cohort, patients with a BASIC score of 2 fared better than those with a BASIC score of 3, with $88 \%$ of BASIC Score 2 patients achieving an AIS grade of C or D and no AIS Grade A at discharge, as opposed to $67 \%$ of BASIC Score 3 patients discharged with AIS Grade A or B (Fig. 5).

Consistent with results based on previous classification systems, ${ }^{3,12,24,27}$ the presence of macroscopic intramedullary hemorrhages in our cohort predicted a poor prognosis. All of the patients with a BASIC score of 4 were

TABLE 3. Principal component analysis of the BASIC score for each rater and the consensus

\begin{tabular}{|c|c|}
\hline Rater No. & Principal Component 1 \\
\hline 1 & 0.966 \\
\hline 2 & 0.988 \\
\hline 3 & 0.769 \\
\hline 4 & 0.971 \\
\hline 5 & 0.845 \\
\hline 6 & 0.915 \\
\hline 7 & 0.947 \\
\hline Consensus score* & 0.988 \\
\hline
\end{tabular}

* The consensus score of 20 images used for the interrater testing was collaboratively arrived at by the 2 developers of the BASIC score scale. 

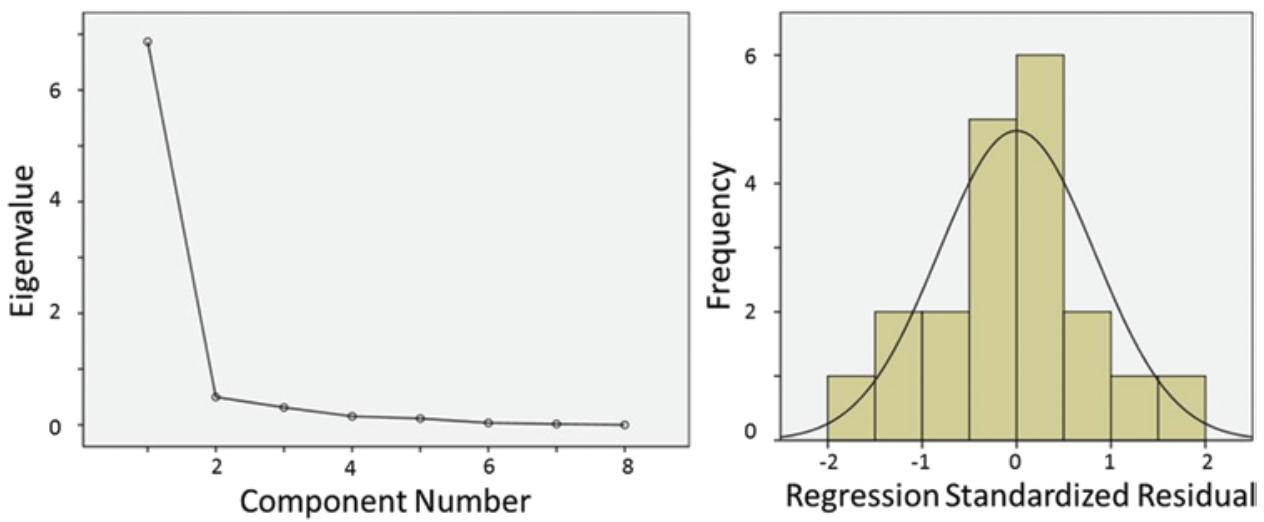

FIG. 7. Left: A screen plot of factor analysis (via principal component analysis extraction) on all ratings, indicating that the BASIC scores for SCls reflect a unidimensional linear metric characterized by a single principal component with an eigenvalue of >1. Right: A principal-component loading matrix indicating that all raters' scores as well as the consensus score loaded very highly onto the BASIC score unidimensional factor (that is, on Principal Component 1). The frequency of residual errors relative to the consensus scores was normally distributed, indicating that novice ratings, on average, strongly and linearly correlated with the expert consensus rating, with only a small number of normally distributed random errors. Together, these results suggest that the BASIC score has high interrater reliability and good parametric properties (see also Table 3). Figure is available in color online only.

discharged with an unchanged AIS Grade A. Of note, we did not use gradient- or susceptibility-weighted sequences, which have demonstrated increased sensitivity to intramedullary blood products. ${ }^{40}$ Further studies are required to evaluate the prognostic value of these more sensitive susceptibility-weighted sequences. Importantly, not all of the patients presenting with AIS Grade A had evidence of macroscopic hemorrhage or diffuse axial T2 hyperintensity. We observed that some patients who presented clinically with complete injury had BASIC scores that suggested a less severe injury (Fig. 6).

Less severe BASIC scores were more commonly observed in those patients with AIS Grade A SCIs that improved by at least one AIS grade by the time of discharge. Thus, the BASIC score discriminated between AIS Grade A patients at presentation whose condition improved by at least one AIS grade by the time of discharge, and those who showed no improvement as assessed by the AIS grading (Fig. 6). While longer-term follow-up and prospective data are needed to corroborate these preliminary results, the present data suggest that the BASIC score may be very helpful in identifying those patients who are the best candidates for clinical trials of experimental higher-risk invasive procedures such as intramedullary injection of stem cells or devices.

The slightly older demographic of the patients in our cohort differs from the typical demographics reported for patients with acute traumatic SCIs. ${ }^{7,34}$ This shift represents a trend we have observed for all SCIs at our institution, with an older second peak in SCI patients after a fall. This appears to reflect the specific population demographic of the San Francisco Bay area. Similar trends have been recently reported in the Canadian population. ${ }^{39}$ Although a demographic subgroup analysis was not performed, no notable differences in patterns of transverse intramedullary T2 signal hyperintensity among age group or injury mechanisms were observed. However, to validate the BASIC score, future studies including larger patient populations across geographic regions are warranted.

\section{Limitations}

There are limitations to the current study, including its retrospective design, variable timing of the acute-phase MRI, and a relatively short clinical follow-up. In addition, interrater reliability testing was not performed directly at a PACS station but rather in a group setting with presentation of index images selected by a neuroradiologist from the injury epicenter and from normal spinal cord. In our opinion, it is in fact easier to assign a BASIC score by scrolling through the axial and sagittal MRI studies on a dedicated PACS station, as is the typical practice followed by most spine surgeons and radiologists. Prospective validation studies with long-term follow-up are planned to validate these preliminary data. An additional limitation is the subjectivity of our classification system. Although qualitative and subjective in nature, the BASIC score scale demonstrated excellent interrater reliability (mean $\kappa$ score $=0.83$ ) across observers with varied expertise. Moreover, it can be performed rapidly without performing manual measurements or time-consuming image postprocessing. Axial T2-weighted imaging is routinely performed as part of MRI protocols for cervical spine trauma and as a recommended sequence for acute spinal cord MRI protocols according to the SCI Common Data Elements of the National Institute of Neurological Disorders and Stroke (NINDS). Therefore, a modification of existing protocols is not required. One limitation of T2 signal-based MRI classification systems such as BASIC for SCI evaluation is the nonspecific nature of the T2 signal hyperintensity. This probably contributes, at least in part, to some of the variable clinical outcomes we observed in patients within each BASIC score group (Fig. 5).

Changes in T2 signals also depend on the timing of the MRI after an injury. ${ }^{23,26,28,38}$ Although we excluded all patients who underwent an MRI examination more than 48 hours after admission and even though $85 \%$ of our patients had MRI within 12 hours of admission, the variable timing of the MRI examination within our selected time interval 
also probably influenced the patterns of observed $\mathrm{T} 2$ signal abnormality in the setting of a rapidly evolving acute SCI. Further studies evaluating the optimal timing of MRI examinations for prognostic purposes during the acute phase of SCI are needed. Despite these limitations and when compared with previous classification systems $3^{3,4,12,35}$ on which it is built, the BASIC score has excellent prognostic capability, particularly for patients with intermediate injury severity. Advanced MRI techniques, including diffusion tensor imaging, magnetization transfer imaging, MR spectroscopy, and functional MRI have shown varying potentials as noninvasive functional biomarkers for SCI. ${ }^{37,41}$ The prognostic superiority of these techniques to standard T2-weighted imaging will need to be established before their routine clinical implementation. The BASIC score for SCIs may represent one standard for such future comparisons.

\section{Conclusions}

We present a novel, simple, and reliable classification system for grading acute blunt traumatic SCIs on the basis of the pattern of $\mathrm{T} 2$ signal abnormality as assessed in the axial plane at the injury epicenter. The BASIC scale has excellent prognostic potential across all SCI severities. These preliminary data suggest that the BASIC score will help distinguish patients who present with an AIS Grade A that improves before discharge from those who will not recover significant function. The proposed classification system builds on the previous literature and may provide prognostic stratification of patients with SCIs by reflecting functionally and anatomically significant patterns of $\mathrm{T} 2$ hyperintensity in the axial plane, which is not dependent on arbitrary measures of longitudinal signal abnormality. Future prospective and well-controlled studies are needed to further validate the prognostic value of the BASIC score.

\section{References}

1. Andreoli C, Colaiacomo MC, Rojas Beccaglia M, Di Biasi C, Casciani E, Gualdi G: MRI in the acute phase of spinal cord traumatic lesions: Relationship between MRI findings and neurological outcome. Radiol Med (Torino) 110:636-645, 2005

2. Basso DM, Beattie MS, Bresnahan JC, Anderson DK, Faden AI, Gruner JA, et al: MASCIS evaluation of open field locomotor scores: effects of experience and teamwork on reliability. Multicenter Animal Spinal Cord Injury Study. J Neurotrauma 13:343-359, 1996

3. Bondurant FJ, Cotler HB, Kulkarni MV, McArdle CB, Harris JH Jr: Acute spinal cord injury. A study using physical examination and magnetic resonance imaging. Spine (Phila Pa 1976) 15:161-168, 1990

4. Bozzo A, Marcoux J, Radhakrishna M, Pelletier J, Goulet B: The role of magnetic resonance imaging in the management of acute spinal cord injury. J Neurotrauma 28:1401-1411, 2011

5. Bresnahan JC, Beattie MS, Todd FD III, Noyes DH: A behavioral and anatomical analysis of spinal cord injury produced by a feedback-controlled impaction device. Exp Neurol 95:548-570, 1987

6. Budde MD, Kim JH, Liang HF, Russell JH, Cross AH, Song SK: Axonal injury detected by in vivo diffusion tensor imaging correlates with neurological disability in a mouse model of multiple sclerosis. NMR Biomed 21:589-597, 2008
7. Burke DA, Linden RD, Zhang YP, Maiste AC, Shields CB: Incidence rates and populations at risk for spinal cord injury: A regional study. Spinal Cord 39:274-278, 2001

8. Chakeres DW, Flickinger F, Bresnahan JC, Beattie MS, Weiss KL, Miller C, et al: MR imaging of acute spinal cord trauma. AJNR Am J Neuroradiol 8:5-10, 1987

9. Collignon F, Martin D, Lénelle J, Stevenaert A: Acute traumatic central cord syndrome: magnetic resonance imaging and clinical observations. J Neurosurg 96 (1 Suppl):29-33, 2002

10. Cotler HB, Kulkarni MV, Bondurant FJ: Magnetic resonance imaging of acute spinal cord trauma: preliminary report. J Orthop Trauma 2:1-4, 1988

11. Flanders AE, Spettell CM, Friedman DP, Marino RJ, Herbison GJ: The relationship between the functional abilities of patients with cervical spinal cord injury and the severity of damage revealed by MR imaging. AJNR Am J Neuroradiol 20:926-934, 1999

12. Flanders AE, Spettell CM, Tartaglino LM, Friedman DP, Herbison GJ: Forecasting motor recovery after cervical spinal cord injury: value of MR imaging. Radiology 201:649655, 1996

13. Goldberg AL, Rothfus WE, Deeb ZL, Daffner RH, Lupetin AR, Wilberger JE, et al: The impact of magnetic resonance on the diagnostic evaluation of acute cervicothoracic spinal trauma. Skeletal Radiol 17:89-95, 1988

14. Gomori JM, Grossman RI: Mechanisms responsible for the MR appearance and evolution of intracranial hemorrhage. Radiographics 8:427-440, 1988

15. Hayashi K, Yone K, Ito H, Yanase M, Sakou T: MRI findings in patients with a cervical spinal cord injury who do not show radiographic evidence of a fracture or dislocation. Paraplegia 33:212-215, 1995

16. Kelley BJ, Harel NY, Kim CY, Papademetris X, Coman D, Wang X, et al: Diffusion tensor imaging as a predictor of locomotor function after experimental spinal cord injury and recovery. J Neurotrauma 31:1362-1373, 2014

17. Kim JH, Loy DN, Liang HF, Trinkaus K, Schmidt RE, Song SK: Noninvasive diffusion tensor imaging of evolving white matter pathology in a mouse model of acute spinal cord injury. Magn Reson Med 58:253-260, 2007

18. Kim JH, Loy DN, Wang Q, Budde MD, Schmidt RE, Trinkaus K, et al: Diffusion tensor imaging at 3 hours after traumatic spinal cord injury predicts long-term locomotor recovery. J Neurotrauma 27:587-598, 2010

19. Kulkarni MV, Bondurant FJ, Rose SL, Narayana PA: 1.5 tesla magnetic resonance imaging of acute spinal trauma. Radiographics 8:1059-1082, 1988

20. Kulkarni MV, McArdle CB, Kopanicky D, Miner M, Cotler HB, Lee KF, et al: Acute spinal cord injury: MR imaging at 1.5 T. Radiology 164:837-843, 1987

21. Landis JR, Koch GG: The measurement of observer agreement for categorical data. Biometrics 33:159-174, 1977

22. Loy DN, Kim JH, Xie M, Schmidt RE, Trinkaus K, Song SK: Diffusion tensor imaging predicts hyperacute spinal cord injury severity. J Neurotrauma 24:979-990, 2007

23. Machino M, Yukawa Y, Ito K, Nakashima H, Kanbara S, Morita D, et al: Can magnetic resonance imaging reflect the prognosis in patients of cervical spinal cord injury without radiographic abnormality? Spine (Phila Pa 1976) 36:E1568E1572, 2011

24. Marciello MA, Flanders AE, Herbison GJ, Schaefer DM, Friedman DP, Lane JI: Magnetic resonance imaging related to neurologic outcome in cervical spinal cord injury. Arch Phys Med Rehabil 74:940-946, 1993

25. Martin D, Schoenen J, Lenelle J, Reznik M, Moonen G: MRI-pathological correlations in acute traumatic central cord syndrome: case report. Neuroradiology 34:262-266, 1992

26. Mihai G, Nout YS, Tovar CA, Miller BA, Schmalbrock P, 
Bresnahan JC, et al: Longitudinal comparison of two severities of unilateral cervical spinal cord injury using magnetic resonance imaging in rats. J Neurotrauma 25:1-18, 2008

27. Miyanji F, Furlan JC, Aarabi B, Arnold PM, Fehlings MG: Acute cervical traumatic spinal cord injury: MR imaging findings correlated with neurologic outcome-prospective study with 100 consecutive patients. Radiology 243:820827,2007

28. Nout YS, Mihai G, Tovar CA, Schmalbrock P, Bresnahan JC, Beattie MS: Hypertonic saline attenuates cord swelling and edema in experimental spinal cord injury: a study utilizing magnetic resonance imaging. Crit Care Med 37:2160-2166, 2009

29. Pearson K: On lines and planes of closest fit to systems of points in space. Philos Mag 2:559-572, 1901

30. Pouw MH, van der Vliet AM, van Kampen A, Thurnher MM, van de Meent H, Hosman AJ: Diffusion-weighted MR imaging within $24 \mathrm{~h}$ post-injury after traumatic spinal cord injury: a qualitative meta-analysis between T2-weighted imaging and diffusion-weighted MR imaging in 18 patients. Spinal Cord 50:426-431, 2012

31. Quencer RM, Bunge RP, Egnor M, Green BA, Puckett W, Naidich TP, et al: Acute traumatic central cord syndrome: MRI-pathological correlations. Neuroradiology 34:85-94, 1992

32. Ramón S, Domínguez R, Ramírez L, Paraira M, Olona M, Castelló T, et al: Clinical and magnetic resonance imaging correlation in acute spinal cord injury. Spinal Cord 35:664673,1997

33. Schaefer DM, Flanders A, Northrup BE, Doan HT, Osterholm JL: Magnetic resonance imaging of acute cervical spine trauma. Correlation with severity of neurologic injury. Spine (Phila Pa 1976) 14:1090-1095, 1989

34. Sekhon LH, Fehlings MG: Epidemiology, demographics, and pathophysiology of acute spinal cord injury. Spine (Phila Pa 1976) 26 (24 Suppl):S2-S12, 2001

35. Shimada K, Tokioka T: Sequential MR studies of cervical cord injury: correlation with neurological damage and clinical outcome. Spinal Cord 37:410-415, 1999

36. Spearman C: General intelligence, objectively determined and measured. Am J Psychol 15:201-293, 1904
37. Stroman PW, Wheeler-Kingshott C, Bacon M, Schwab JM, Bosma R, Brooks J, et al: The current state-of-the-art of spinal cord imaging: methods. Neuroimage 84:1070-1081, 2014

38. Sun LQ, Shen Y, Li YM: Quantitative magnetic resonance imaging analysis correlates with surgical outcome of cervical spinal cord injury without radiologic evidence of trauma. Spinal Cord 52:541-546, 2014

39. Thompson C, Mutch J, Parent S, Mac-Thiong JM: The changing demographics of traumatic spinal cord injury: An 11-year study of 831 patients. J Spinal Cord Med 38:214-223, 2015

40. Wang M, Dai Y, Han Y, Haacke EM, Dai J, Shi D: Susceptibility weighted imaging in detecting hemorrhage in acute cervical spinal cord injury. Magn Reson Imaging 29:365373, 2011

41. Wheeler-Kingshott CA, Stroman PW, Schwab JM, Bacon M, Bosma R, Brooks J, et al: The current state-of-the-art of spinal cord imaging: applications. Neuroimage 84:1082-1093, 2014

42. Wilson JR, Cadotte DW, Fehlings MG: Clinical predictors of neurological outcome, functional status, and survival after traumatic spinal cord injury: a systematic review. J Neurosurg Spine 17 (1 Suppl):11-26, 2012

\section{Author Contributions}

Conception and design: Dhall, Talbott. Acquisition of data: Dhall, Talbott, Whetstone, Readdy. Analysis and interpretation of data: Dhall, Readdy, Mabray. Drafting the article: Talbott. Critically revising the article: Dhall, Whetstone, Ferguson, Bresnahan, Beattie, Pan, Manley. Reviewed submitted version of manuscript: Bresnahan, Beattie, Pan, Manley, Saigal, Hawryluk. Statistical analysis: Ferguson. Study supervision: Dhall.

\section{Correspondence}

Sanjay S. Dhall, Department of Neurological Surgery, University of California, San Francisco, 505 Parnassus Ave., Rm. M-779, San Francisco, CA 94143. email: sanjay.dhall@ucsf.edu. 